\title{
Inherent directionality explains the lack of feedback loops in empirical networks
}

SUBJECT AREAS:

COMPUTATIONAL

SCIENCE

COMPLEXITY

Received

11 August 2014

Accepted

24 November 2014

Published

22 December 2014

Correspondence and requests for materials should be addressed to M.A.M. (mamunoz@ onsager.ugr.es)

\author{
Virginia Domínguez-García', Simone Pigolotti ${ }^{2}$ \& Miguel A. Muñoz'
}

'Departamento de Electromagnetismo y Física de la Materia, and Instituto Carlos I de Física Teórica y Computacional Universidad de Granada, 18071 Granada, Spain, ${ }^{2}$ Departament de Fisica i Enginyeria Nuclear, Universitat Politecnica de Catalunya Rambla Sant Nebridi 22, 08222 Terrassa, Barcelona, Spain.

We explore the hypothesis that the relative abundance of feedback loops in many empirical complex networks is severely reduced owing to the presence of an inherent global directionality. Aimed at quantifying this idea, we propose a simple probabilistic model in which a free parameter $\gamma$ controls the degree of inherent directionality. Upon strengthening such directionality, the model predicts a drastic reduction in the fraction of loops which are also feedback loops. To test this prediction, we extensively enumerated loops and feedback loops in many empirical biological, ecological and socio-technological directed networks. We show that, in almost all cases, empirical networks have a much smaller fraction of feedback loops than network randomizations. Quite remarkably, this empirical finding is quantitatively reproduced, for all loop lengths, by our model by fitting its only parameter $\gamma$. Moreover, the fitted value of $\gamma$ correlates quite well with another direct measurement of network directionality, performed by means of a novel algorithm. We conclude that the existence of an inherent network directionality provides a parsimonious quantitative explanation for the observed lack of feedback loops in empirical networks.

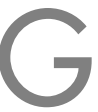
enetic regulatory circuits, metabolic pathways, food webs, and many different socio-technological systems can be visualized as networks made up of units linked pairwise whenever there is some sort of "interaction" or "flow" between them. In many cases, empirical networks are dynamical, time-changing entities, and most of the existing compiled datasets represent static snapshots or time-averages over some observation interval of these more complex processes. Nevertheless, the description in terms of static networks has proven to be useful to identify structural features which are responsible for emerging functions ${ }^{1-4}$. Some structural features, including clustering, degree assortativity ${ }^{5}$, and the relative abundance of specific motifs ${ }^{6,7}$, characterize the topology at the local scale. Other traits, such as nestedness ${ }^{8,9}$, community structure ${ }^{10,11}$, and the existence of a hierarchy ${ }^{12,13}$ are related to the large-scale organization. Clearly, these features are not necessarily independent.

In many empirical networks, interactions are directed, i.e. links have an origin and a target node. This direction can be generally thought of in terms of flows, such as the energy transfer in food webs ${ }^{14}$ and the flow of biological information in genetic or neural networks. Often, this flow identifies a global inherent directionality. By "inherent directionality" we mean that all nodes can be ordered on a one-dimensional axis, in such a way that links point preferentially from low to high values of their coordinates in such an axis. In this sense, the existence of an inherent directionality is deeply related to the existence of a hierarchical structure ${ }^{13,15}$. For example, $(i)$ in networks where there is a transfer of matter, such as food webs or metabolic networks, one can identify a hierarchy of "trophic" levels (links tend to point from lower levels to higher ones), (ii) in gene regulatory networks there is a hierarchy of control (controller nodes act upon controlled ones), and (iii) in neural networks, the flow of information propagates from sensory neurons at the bottom of the hierarchy, to neurons in the central system at intermediate levels, and from there to the level of motor neurons.

The existence of an inherent directionality can have a deep impact on the network small-scale structure, in particular on the statistics of motifs, such as feedback loops. In a directed network, a "feedback loop" of length $k$ is defined as a closed sequence of $k$ different nodes in which a walker following the directions of the arrows returns to the starting point after visiting once and only once all $k$ nodes. Feedback loops are well-known to have a profound impact on dynamical stability in food webs ${ }^{16-23}$ as well as in biological and generic networks ${ }^{7,24-35}$. "Structural loops" or simply "loops", defined as closed sequences of pairwise connected nodes, independently of the direction of links are also of interest. Clearly, the set of feedback loops is a subset of that of structural loops.

The relationship between the existence of a inherent directionality and feedback loops can be intuitively understood by considering the case of perfect directionality -or feedforwardness- in which all links are aligned 
with the inherent directionality. In such perfectly directional networks, feedback loops are completely absent, as at least one link against the directionality is required to close a feedback loop. The impact of directionality on the statistics of feedback loops is less trivial to assess in cases of incomplete feedforwardness, where directionality only partially determines the direction of links.

In this paper, we present a simple model relating an assumed degree of inherent directionality with the statistics of feedback loops in networks. Our model depends on a single parameter, $\gamma$, defined as the probability of any link in the network to point along the inherent direction (see Fig. 1). An analytical calculation allows us to predict the fraction $F(k)$ of loops of length $k$ which are feedback loops. We show that, as long as there exist a inherent directionality, i.e. as long as $\gamma \neq 1 / 2$, the fraction of feedback loops $F(k)$ of any loop lengths $k-$ for which we provide analytical estimations- is much smaller than it would be in network randomizations.

To test the model predictions against empirical data, we scrutinize a number of empirical biological, ecological, and also sociotechnological directed networks. For each of these empirical networks, we perform an extensive computational study of the number of structural and feedback loops it includes. In nearly all the networks we analyzed, we find that $F(k)$ is dramatically smaller than in randomizations of the same networks. Remarkably, the model reproduces the curves $F(k)$ with good precision for all the empirical networks we studied, just by fitting its only free parameter, quantifying the degree of inherent directionality.
Furthermore, we introduce a method to directly estimate the degree of directionality in any given network by employing topological information only. The resulting measurement for each specific network correlates quite well with the directionality parameter employed to obtain the fit for the statistics of feedback loops. We also verify that our results are robust against network subsampling or lack of knowledge of existing connections. Therefore, we conclude that the lack of feedback loops stems from the existence of a inherent directionality in empirical networks.

\section{Results}

Counting loops in empirical networks. We analyzed a large set of empirical biological, ecological and socio-technological directed networks taken from the literature (for the complete list see Supplementary Information S1). We excluded from our analyses un-directed networks and tree-like networks with no single loop of any size. Self-loops -being unrelated to inherent directionality- have not been taken into account. For each network and each loop-length $k$, we exhaustively counted the number of structural loops and the fraction of them which are also feedback loops, $F(k)$. We remark that knowledge of the hierarchical level of each node (if any) is not necessary for this computation.

From a computational perspective, counting loops is a nonpolynomial (NP) hard problem, thus becoming an unfeasible task for large network sizes. For this reason, previous studies often used less computationally-expensive proxies -such as the Estrada index ${ }^{36}$
A

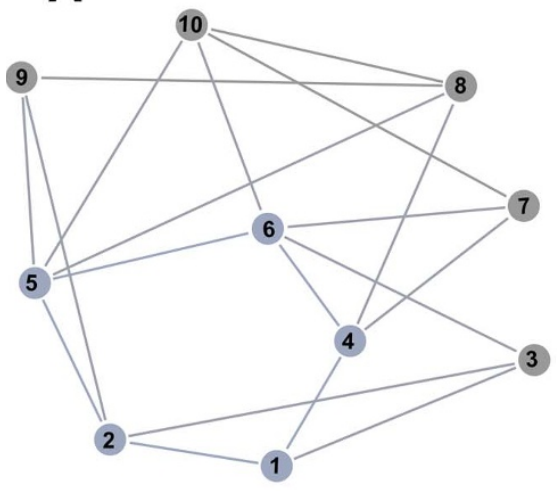

EMPIRICAL NETWORK

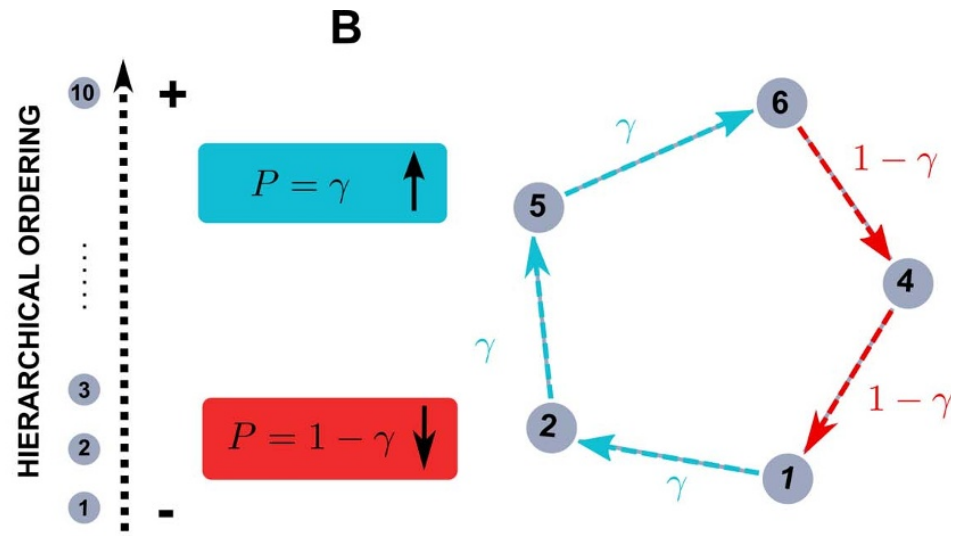

IMPOSED INHERENT DIRECTIONALITY

C
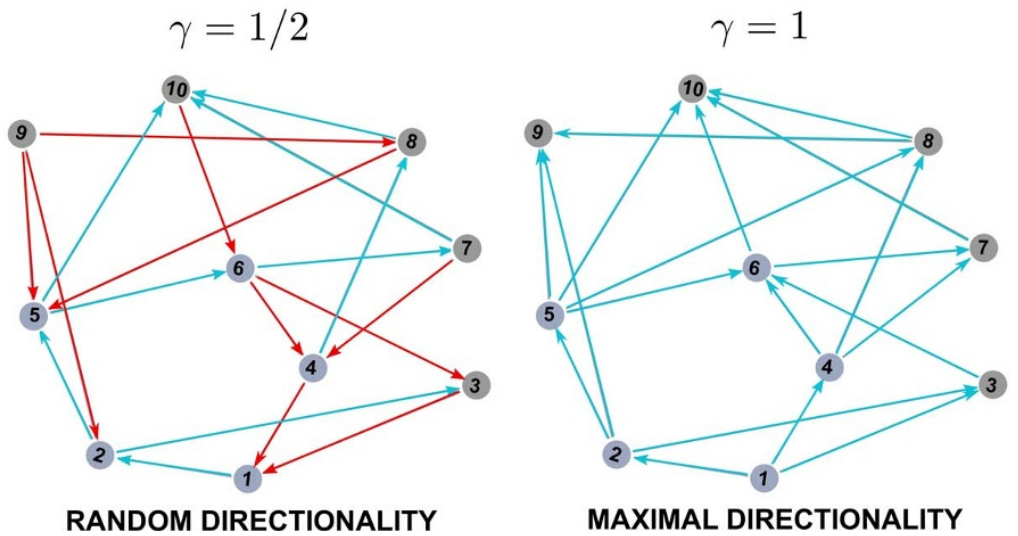

Figure 1 Schematic representation of the directionality model. (A) A network in which nodes are labeled according to some existing inherent ordering or hierarchy, which identifies an inherent directionality. (B) In any given feedback loop, arrows point in the direction of increasing labels, i.e. along the inherent directionality, with probability $\gamma$ (blue arrows) or against it with probability $1-\gamma$ (red arrows). (C) Example of networks with $\gamma=1 / 2$ (random directionality) and with $\gamma=1$ (perfect or maximal directionality). 
or analytical estimations for large network sizes ${ }^{37}$ - to estimate the amount of loops in empirical networks. Despite the non-polynomial nature of the problem, present computer power allows us to count loops up to reasonably-large sizes by using an efficient breadth first algorithm (see Supplementary S2 for more information on the algorithm).

We compared the measured fraction of feedback loops $F(k)$ with two different randomizations of the same network. The first one that we term directionality randomization (DR)- preserves the existing links, but fully randomizes their directions. The second one configuration randomization (CR) - randomizes both links and directions, but preserving the in and out connectivity of each single node $^{38}$ (see Supplementary S3).

Our results, shown in Fig. 2, exhibit a clear trend: the fraction of feedback loops of any length $k$ is much smaller in biological and ecological networks than would be expected for any of the two different randomizations. Let us caution that randomly wired networks of finite size can exhibit small statistical deviations from the largesize limit $\gamma=1 / 2$.

The total number of feedback loops - not just its fraction- is also severely reduced with respect to network randomizations in all the considered biological and ecological networks, as firstly noted in ref. 39 (see Supplementary Fig. S2). These trends are not so evident for socio-technological networks; while all of the considered networks have a smaller fraction of feedback loops than their directionality randomizations, some of the social ones (e.g. "twitter followings" and "political blogosphere") have a larger $F(k)$ than configurational randomizations.

We now test the predictions of our probabilistic model against the empirically measured values of $F(k)$ in all empirical networks. For each of the analyzed empirical networks we consider loop lengths ranging from $k=3$ to maximum values up to $k=12$, determined by computational capabilities and depending crucially on network size and connectivity. For each network, we estimate the value of the directionality parameter $\gamma$ which best describes the observed fraction of feedback loops via an unweighted least-square fit of $\log F(\gamma, k)$ as a function of $k$.

Results are summarized in Fig. 2. The model reproduces remarkably well empirical data for all loop lengths by fitting the only free parameter. In some cases, such as for the neural connectivity (C. elegans) network, the agreement between empirical data and model predictions is quite impressive, while significant deviations are observed in some other cases for small loop lengths, $k \leq 4$. In particular, the worst agreement is obtained for the Coachella valley foodweb. However, this network, with only 29 nodes, is the smallest in the dataset, so that it can deviate significantly from statistical predictions and it has been previously reported to be anomalous from other viewpoints ${ }^{40}$. In some cases, such as the N.E. Shelf foodweb and the two considered transcription regulatory networks ( $E$. coli TRN and Yeast TRN), $\gamma>0.999$ indicating a rather extreme level of inherent directionality (see Table 1). We obtained similar results for other empirical networks with very few loops (listed in Table 1 as well), providing additional support to our conclusion.

As the model predicts an asymptotic exponential decay of $F(k)$ as the loop-length $k$ increases, we have performed -for each particular network- a fit of the empirical data (for $k>4$ ) to an exponential function (see dashed red lines in Fig. 2). In this case, the quality of the fit of $\log F(k)$ versus $k$ can be assessed via a linear regression coefficient, $r$. Obtained values of $r^{2}$ (Table 1) are larger than 0.99 in all cases except one -the Mammalian cell signaling network, for which $r^{2}=0.973-$ indicating that even for relatively small loop-lengths the predicted asymptotic exponential decay holds. Furthermore, each of these exponential fits is very close to its corresponding analyticallyobtained asymptotic result, Eq. (4) (blue discontinuous lines in Fig. 2). In the few cases in which the analytical asymptotic prediction breaks down (see Methods) the blue dashed lines correspond to a fit of the model data for $k \leq 4$. This shows that the asymptotic expression is reasonably accurate even for rather short loops.

We conclude this section with a remark on the possible impact of unknown links. Our knowledge of biological and technological networks is often incomplete and it is important to assess how this fact may affects our analyses. To test the robustness of our framework, we mimicked the effect of undersampling of empirical networks by eliminating a fraction of the links at random, and repeated the analysis above. While this operation clearly affects the number of links, the conclusions of our model (in particular the fitted value of $\gamma$ ) are very weakly modified even when a relatively large fraction of nodes $(20 \% \sim 50 \%)$ is removed. Details are presented in Supplementary Information S5 and Supplementary Fig. S3.

Measuring the degree of directionality of empirical networks. The directionality parameter $\gamma$ in the probabilistic model represents how strongly the hypothesized hierarchical ordering affects the direction of the links in the network; $\gamma=1$ (and also $\gamma=0$ ) reflect perfect directionality while $\gamma=1 / 2$ corresponds to random directionality. In the previous section, $\gamma$ has been inferred from the statistics of feedback loops.

We now propose an algorithm to directly measure the degree of directionality of a network from its topology. Similar methods have been proposed for this purpose ${ }^{41-43}$. All of them are able to extract a hierarchical ordering from a given network and classify nodes into a few discrete levels. Instead, the method we propose produces more refined orderings, being able to resolve possible degeneracies between the coarser levels produced by previous methods (see ref. 44).

Our method is inspired by algorithms for determining trophic levels in food webs, but is applicable to any directed network; it can be also seen as a way to infer a "hidden variable" from network topology ${ }^{45}$. As customarily done with food webs, one identifies "basal nodes" as those having zero in-connectivity, i.e. with no link pointing to them. In the possible case in which no basal node exists, we progressively identify sets made out of two, three... nodes which taken as a unique coarse-grained node- are basal, i.e. no external node points to any node in the set.

Basal nodes obtained in this way are placed at the lowest level of the hierarchical ordering, $l=0$. Then, the level of the remaining nodes is defined as the average of the trophic level of all nodes pointing to it (its preys in food webs) plus 1 :

$$
l_{j}=1+\frac{1}{k_{j}} \sum_{i} A_{i j} l_{i},
$$

where $k_{j}$ is the in-connectivity of node $j, A_{i j}$ is the connectivity or adjacency matrix and $l_{j}$ is the hierarchical level of node $j$. The conditions (1) define a set of linear equations in the unknown $l_{j}$ 's that can be solved using standard algebraic methods. Notice that, while with existing methods ${ }^{41-43}$ hierarchical levels associated to nodes are integer numbers, here they are in general real numbers. Further details, examples and applications of this method will be published elsewhere.

Using the hierarchical ordering resulting from applying the algorithm above, it is straightforward to compute the fraction of links pointing from lower to higher hierarchical levels, i.e. aligned with the inherent directionality. We call this fraction "current parameter", $\chi$. In the limit of perfect feedforwardness one expects $\chi=1$, while in the absence of a well-defined directionality $\chi \approx 1 / 2$ (apart from small deviations due to finite-size effects).

Our results are summarized in Fig. 3. They clearly show that all the considered biological, ecological, and also -to much lesser extentsocio-technological networks exhibit some degree of hierarchy, $\chi>$ $1 / 2$. More remarkably, the explicitly measured values of $\chi$ correlate quite well with the fitted value of the directionality parameter $\gamma$ in the set of networks under study. This correlation implies that the free 
E. Coli TRN

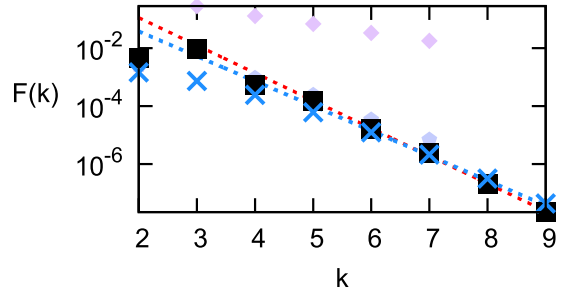

Neural Connectivity

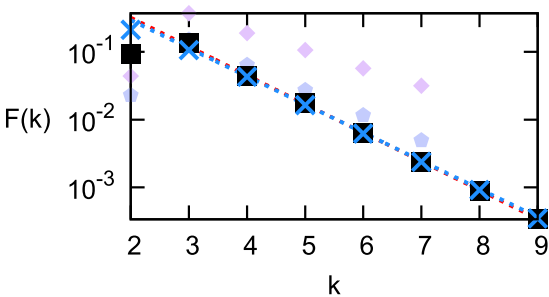

EI Verde Rainforest

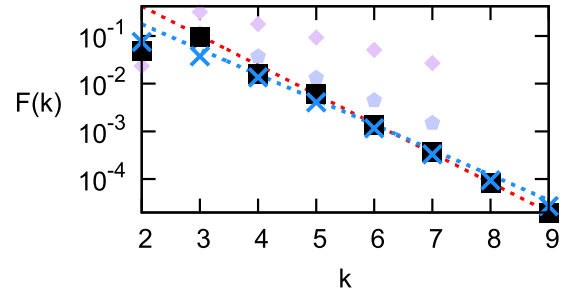

B

F.A.A

$\mathrm{F}(\mathrm{k})$

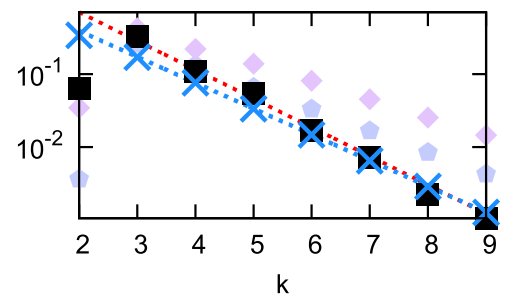

Political Blogosphere

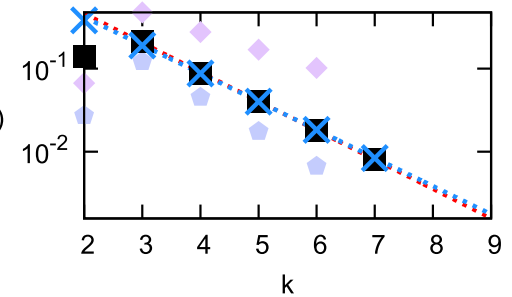

Wikipedia elections

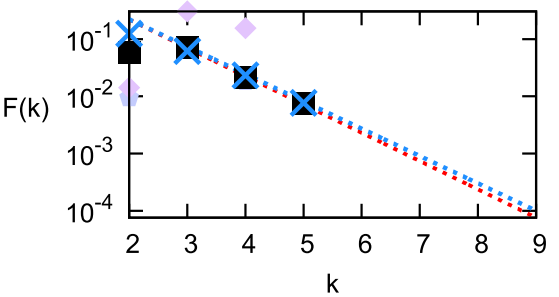

Cell Signaling

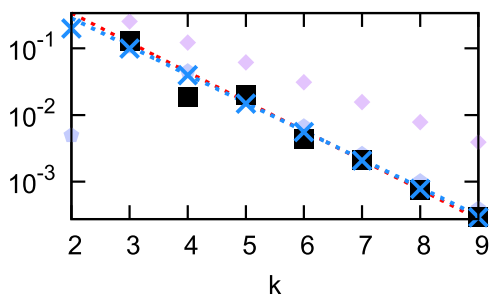

Coachella Valley

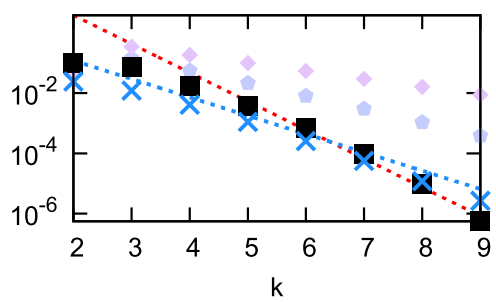

N.E. Shelf

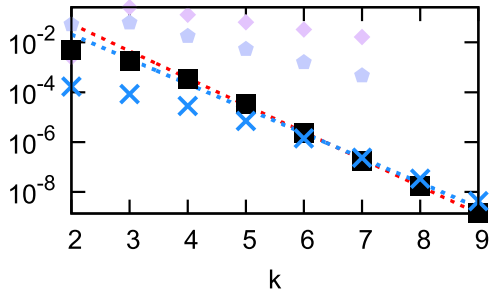

Forum Replies

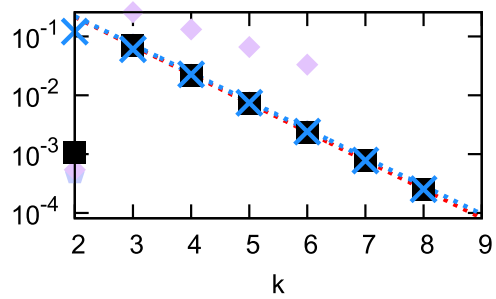

Advocato Forum

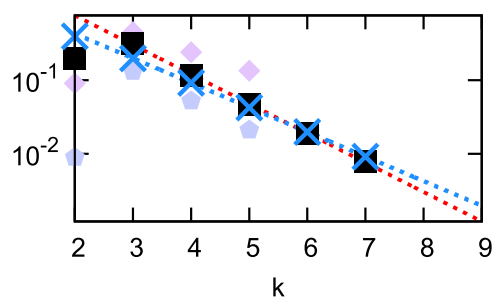

P2P Conections

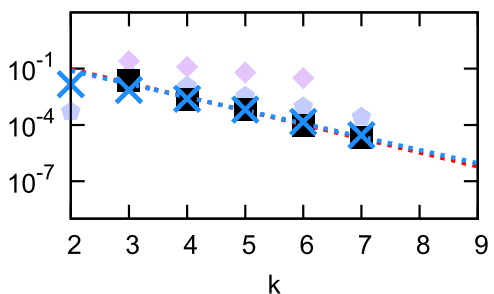

Yeast TRN

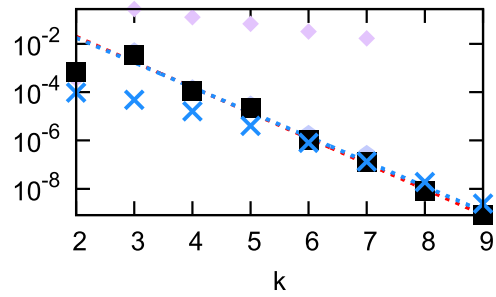

Caribbean Reef

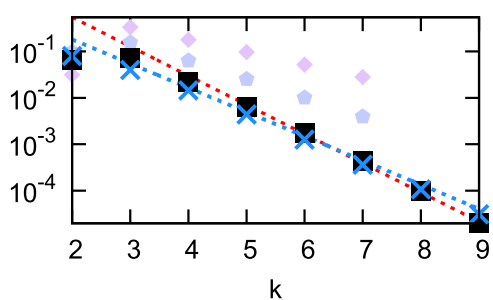

Little Rock Lake

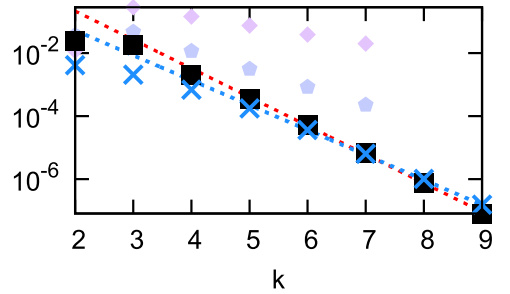

Twitter Followings

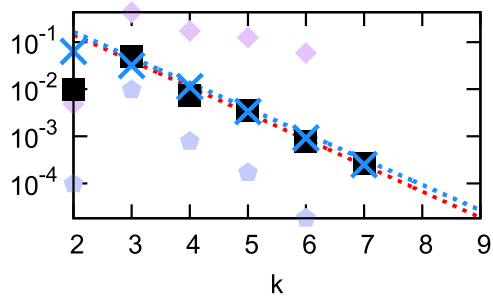

Kaitiaki frienship

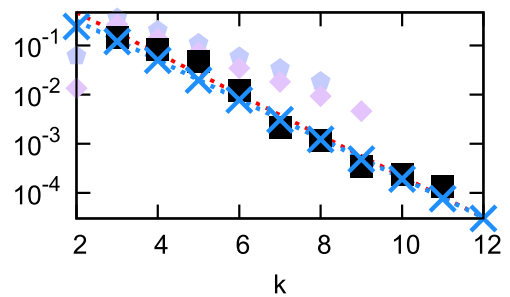

Figure $2 \mid$ Fraction of feedback loops, $F(k)$, as a function of the loop length, $k$, in empirical networks. Black squares correspond to empirical data and red dashed lines stand for fits of the empirical data to an asymptotic exponential curve (fit done using data for $k>4$ ). Pale blue pentagons stand for configurational randomizations and pale pink diamonds for directionality randomizations. Blue crosses mark the best fit of our probabilistic model (the parameter $\gamma$ has been fitted using a least-squares method to $\log F(k)$ versus $k$ ). The resulting optimal $\gamma$ values for the different networks are compiled in Table 1. Blue dashed lines correspond to the asymptotic analytical estimate of Eq.(4) for the corresponding $\gamma$. Notice the closeness between the exponential fit to empirical data and the analytical prediction. 
Table 1 | Quantification of network directionality. First and second columns: values of the linear correlation coefficient $r^{2}$ and of the fitted parameter $\gamma$, respectively, for the linear fit of $\log F(k)$ versus $k$ with Eq. (3) for the considered networks. Third column: measures of the current parameter $\chi$ from the network structure (large values of $\chi$ indicate high levels of hierarchy and thus of directionality). Networks below the central double line are those with only a small number of short loops, i.e. not having any loop larger than $k=6$. In the case of the Skipwith network, the value of $r^{2}$ is absent as we could not compute long enough loops to observe the exponential decay. In the Twitter followings network the value of $\chi$ could not be computed due to computational limitations

\begin{tabular}{lccc} 
Network & $r^{2}$ & $\gamma$ & $\chi$ \\
\hline E. coli TRN & 0.995 & 0.999 & 0.9316 \\
Cell Signaling & 0.973 & 0.888 & 0.7348 \\
Yeast TRN & 0.997 & 1.000 & 0.9887 \\
Neural Connectivity & 1.000 & 0.879 & 0.7429 \\
Coachella Valley & 0.984 & 0.988 & 0.7325 \\
Caribbean Reef & 0.997 & 0.958 & 0.8579 \\
El Verde Rainforest & 0.997 & 0.961 & 0.8381 \\
N.E. Shelf & 0.997 & 1.000 & 0.9470 \\
Little Rock Lake & 0.999 & 0.998 & 0.9350 \\
Lough Hyne & 0.980 & 0.999 & 0.9616 \\
Weddell Sea & 0.992 & 0.987 & 0.9072 \\
F.A.A. & 0.998 & 0.783 & 0.6183 \\
Forum Replies & 1.000 & 0.935 & 0.7359 \\
Twitter Followings & 0.984 & 0.967 & - \\
Political Blogosphere & 1.000 & 0.744 & 0.5217 \\
Advocato Forum & 0.999 & 0.714 & 0.5487 \\
Kaitiaki friendship & 0.975 & 0.860 & 0.8411 \\
Wikipedia elections & 0.998 & 0.934 & 0.7252 \\
P2P Connections & 0.991 & 0.993 & 0.8205 \\
Everglades & 0.979 & 0.999 & 0.9673 \\
Mangrove Estuary & 0.998 & 0.999 & 0.9704 \\
Mondego Estuary & 1.000 & 0.992 & 0.9373 \\
Skipwith & 0.000 & 0.997 & 0.9471 \\
Human TRN & 0.984 & 0.999 & 0.9626 \\
Mouse TRN & 1.000 & 0.970 & 0.8957 \\
Ownership & 1.000 & 0.977 & 0.9880 \\
Tuberculosis TRN & 1.000 & 0.998 & 0.9858 \\
B. subtilis TRN & 1.000 & 0.985 & 0.9459 \\
& & & \\
& & &
\end{tabular}

parameter we use to fit the directional model is consistent with a direct measure of directionality (current) in the same networks.

\section{Discussion}

While the crucial role of feedback loops in determining dynamical properties of complex networks has been widely recognized in the literature, their statistics remained scarcely studied. Some exceptions are Refs. 46, 47 where, respectively, the under-representation of long feedback loops in the $E$. coli gene regulatory network and the overrepresentation of short feedback loops in the S. cerevisae's one were first noticed, as well as ref. 39 where the statistics of the total number of feedback loops in complex networks was studied.

We have tackled the problem of exhaustively counting the number of structural loops and feedback loops in a variety of biological, ecological, and socio-technological networks. We then compared these numbers with those in randomized versions of the same graphs, where other basic structural features (such as total number of nodes, number of links, connectivity of each link, etc.) were preserved. In all the analyzed biological and ecological networks we find a dramatic reduction of the fraction of loops which are also feedback loops with respect to random expectations. This effect is much milder in sociotechnological networks.

We hypothesize that the (empirically observed) lack of feedback loops stems from the existence of an inherent directionality. To investigate this conjecture, we have constructed a simple computational model in which an inherent network directionality -quantified by a directionality parameter $\gamma$ - is built in. For this model we are able to analytically compute the fraction of feedback loops of any given length as a function of $\gamma$. Our main result is that this intrinsically directional model can reproduce quite well empirical curves of the fraction of feedback loops of any length by just tuning its only parameter $\gamma$. For example, for some networks such as the neural connectivity network, empirical results fall in a nearly-perfect way on top of the model curve for all loop-lengths with amazing accuracy. The quality of the results is even more remarkable if we consider that our model assumes a number of simplifications that are by no means trivial. For instance, the model neglects any correlation or relation among different loops: each loop is treated separately, while in empirical networks, especially if they have broad connectivity distribution functions, typically loops are not independent as they can share some nodes. In particular, hubs are statistically more likely than other nodes to take part in loops. Furthermore, node degree and position in the network hierarchy could well be correlated in empirical networks, while such an hypothetical correlation is just neglected by our simple model. These effects could be responsible for the small departures of empirical data from our model predictions.

It is even more remarkable that the optimal value of the directionality parameter $\gamma$-derived from the statistics of loops- correlates quite well with the current parameter, $\chi$, computed by quantifying the network "stratified" architecture or degree of directionality. These two measures of network inherent directionality are quantitatively different but they are strongly correlated.

It is interesting to recall that the first model of food web architectures ${ }^{48}$ did include a perfect directionality and thus complete absence of feedback loops, while more recent models (see e.g. refs. 49, 50, 51) allow for some small degree of backward edges, enabling directed loops to appear.

Our finding is similar in spirit to the remarkable observation by Mayaa'n et al. that biological networks display a kind of antiferromagnetic ordering - meaning that contiguous links have a statistical tendency to point in opposite directions- causing a depletion of feedback loops which they claim lead to an enhancement of network stability $^{52}$. Instead, our hypothesis here is that the absence of feedback loops is a byproduct of a more inherent feature of networks: the existence of a preferred directionality. Indeed, by employing a method inspired on how trophic levels are identified in food webs, we have been able to identify -just by looking at the network structure- an objectively measured correlate of the fitted directionality parameter. Similarly, in a recent work, it is claimed that long loops are over-represented in biological networks ${ }^{53}$. The origin of the apparent conflict with our results can be tracked down to the different definition of loops employed in ref. 53, where only "minimal loops" (see ref. 53 for a definition) are considered rather than the exhaustive enumeration of all loops we perform here.

Summarizing, our results show that the existence of an inherent directionality constitutes a simple yet satisfactory parsimonious explanation for the empirically observed lack of feedback loops in biological and ecological networks.

\section{Methods}

Network directionality model. Let us consider a network consisting of $N$ nodes and $L$ directed links and imagine that the fraction of loops which are also feedback loops, $F(k)$, is known. We now aim at constructing a probabilistic model able to predict the empirically-measured function $F(k)$. The model consists in taking the empirical network under consideration and randomizing the direction of each single link with the constraint that some degree of inherent directionality exists. We therefore assume that nodes can be characterized by an index or coordinate $i=1 \ldots N$ representing their position along the directionality axis. As a convention, we choose higher nodes in the hierarchy to have larger labels, as shown in Fig. 1A. A direction to each existing link is (re-)assigned as follows (see Fig. 1B): a link is set to point from a lower label to the higher one, with probability $\gamma$, where the "directionality parameter" $\gamma$ satisfies $0 \leq$ 


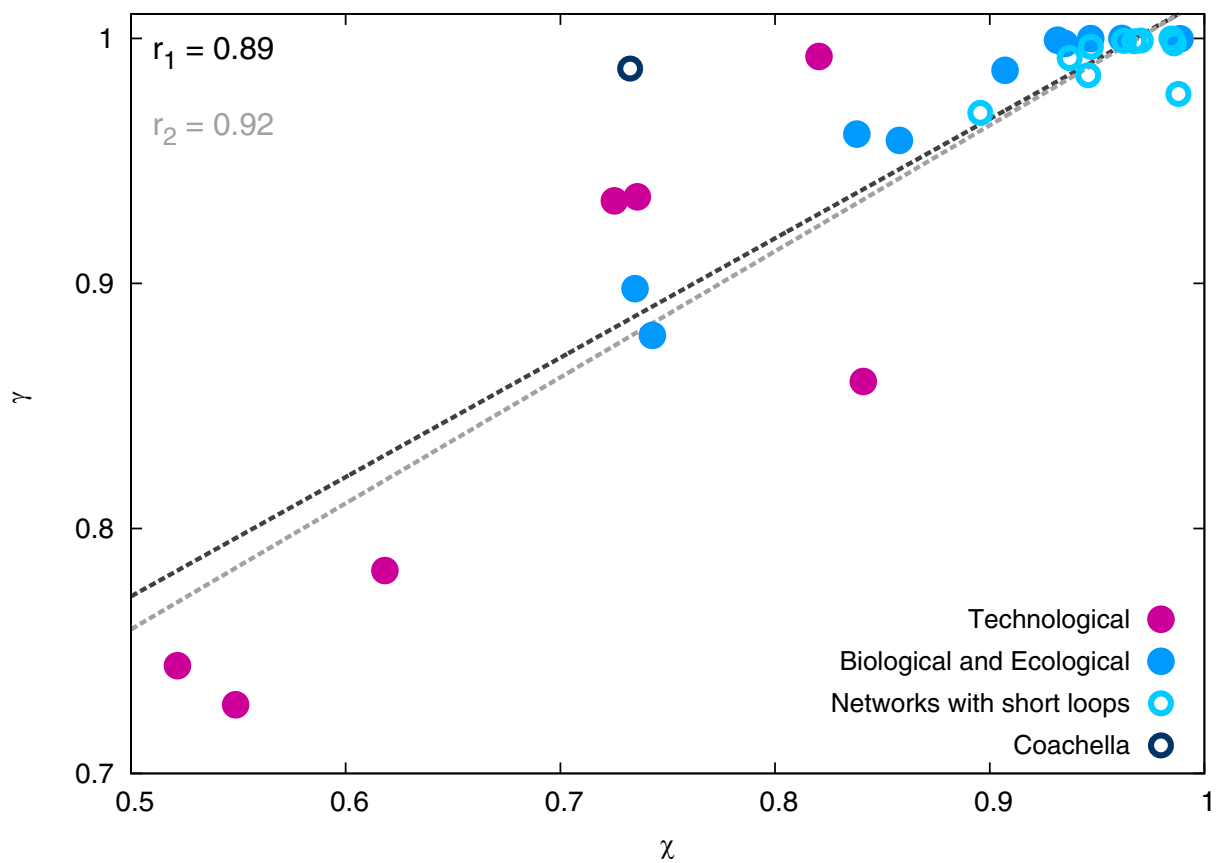

Figure 3 Correlation between inferred and explicitly measured levels of directionality. Scatter plot of the optimal values of the directionality parameter $\gamma$ plotted against the current parameter $\chi$. Values of either $\gamma$ or $\chi$ close to 1 reflect a high degree of directionality while smaller values close to $1 / 2$ imply that link directions are nearly uncorrelated with directionality. The value of the linear correlation coefficient is $r=0.89$ or $r=0.92$ depending on whether the outlier "Coachella Valley" small network is included or not. The corresponding best fits are $\gamma=0.487 \chi+0.529$ and $\gamma=0.514 \chi+0.502$, respectively.

$\gamma \leq 1$. With the complementary probability $1-\gamma$ the link points against the inherent directionality. In particular, $\gamma=1$ (or $\gamma=0)$ stands for perfect inherent directionality, while for $\gamma=1 / 2$, the inherent directionality does not affect the direction of the links.

Our goal is to analytically estimate the expected value of $F(k)$ for any given loop length $k$ as a function of the only parameter. To make progress, we consider loops independently, i.e. we neglect possible correlations between for example loops having common links in a same network. We also neglect the impact of possible heterogeneities in the distribution of loops across hierarchical levels. In the case of empirical networks we are interested in, we shall assume these as working hypotheses, whose validity will be tested $a$ posteriori by comparing our results against data.
Under these assumptions, we focus on a specific loop of arbitrary length $k$ (see Fig. 1). Without loss of generality, we re-label the node indexes onto the integer numbers $1 \ldots k$ by preserving the ordering, i.e. we label the node having the lowest index in the loop with 1 , the second lowest with 2 and so on. In this way, the loop is associated with a permutation $\{n\}=n_{1}, n_{2} \ldots n_{k}$, where $n_{i}$ is the label of the $i-t h$ node in the loop. Formally, we define $n_{k+1}=n_{1}$ to ensure that the loop is closed.

Under the assumptions above, we consider that all the $k$ ! possible loop permutations are equally likely to be found. In this way, the maximum number of feedback loops is expected to occur for $\gamma=1 / 2$, for which the two directions are equi-probable. In this case, $F(k)=2^{1-k}$ as only 2 out of the possible $2^{k}$ loops of length $k$ are feedback

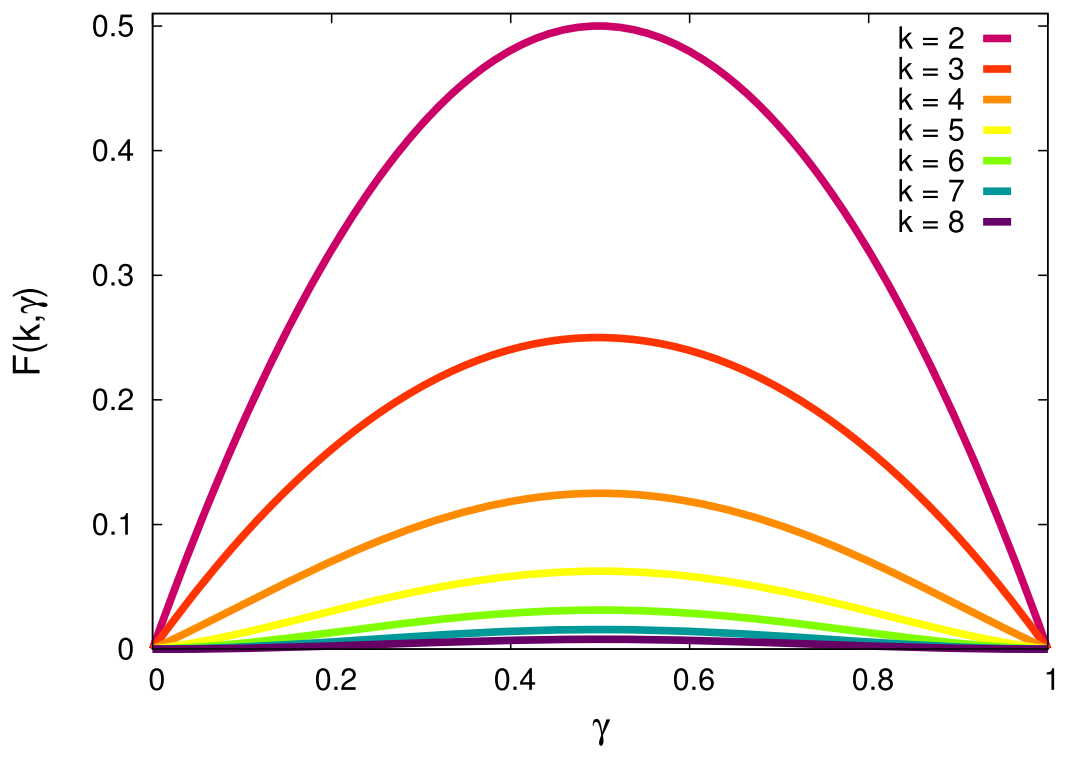

Figure $4 \mid$ Fraction of feedback loops, $F(\boldsymbol{k})$, versus the directionality parameter $\gamma \cdot F(k)$ has a maximum at $\gamma=1 / 2$, for which all link directions are randomly set, giving rise to the largest possible fraction of directed loops. On the other side, $F(k)$ vanishes for $\gamma=0$ and for $\gamma=1$ as expected. Notice also that the curves are symmetric around $\gamma=1 / 2$ and that for values of $\gamma$ different from $1 / 2$ one has a directionality-induced lack of feedback loops. 


\begin{tabular}{|c|c|c|c|c|c|c|c|c|c|}
\hline kl & 0 & 1 & 2 & 3 & 4 & 5 & 6 & 7 & 8 \\
\hline 1 & 1 & & & & & & & & \\
\hline 2 & & 2 & & & & & & & \\
\hline 3 & & 3 & 3 & & & & & & \\
\hline 4 & & 4 & 16 & 4 & & & & & \\
\hline 5 & & 5 & 55 & 55 & 5 & & & & \\
\hline 6 & & 6 & 156 & 396 & 156 & 6 & & & \\
\hline 7 & & 7 & 399 & 2114 & 2114 & 399 & 7 & & \\
\hline 8 & & 8 & 960 & 9528 & 19328 & 9528 & 960 & 8 & \\
\hline 9 & & 9 & 2223 & 38637 & 140571 & 140571 & 38637 & 2223 & 9 \\
\hline
\end{tabular}

loops. In a more general case, the probability of a given loop to be a feedback loop depends on the distribution of the number of ascents, i.e. the number $A(l, k)$ counting how many permutations of the basic sequence of length $k$ are such that $n_{i}<n_{i+1}$ holds for exactly $l$ distinct values of $i$. For a non-periodic sequence, i.e. without establishing any relation between $n_{k}$ with $n_{1}$, the solution to this problem is given by the so-called Eulerian numbers (see e.g. ref. 54 chapter 6 or ref. 55). Since loops are closed, we need to generalize the concept of Eulerian numbers to the periodic or cyclic case, i.e. we need to count the number of ascents in a generic closed loop, which we call "cyclic Eulerian numbers", $A(l, k)$. Further in this section we prove a recursion relation

$$
(k-1) A(l, k)=k[(k-l) A(l-1, k-1)+l A(l, k-1)]
$$

which generalizes a similar relation for standard Eulerian numbers (see e.g. ref. 54) and which allows us to recursively find all cyclic Eulerian numbers. Notice, in particular, that $A(0, k)=A(k, k)=0 \mu k$ as it is clearly impossible to have all ascents/ descent in a closed loop. Examples of cyclic Eulerian numbers for values of $k$ up to 9 are also presented later in Methods.

The expected fraction $F(k, \gamma)$ of loops of length $k$ which are feedback loops can be expressed as

$$
F(k, \gamma)=\sum_{l=0}^{k} \frac{A(l, k)}{k !}\left[\gamma^{l}(1-\gamma)^{k-1}+\gamma^{k-l}(1-\gamma)^{l}\right]
$$

where the two terms in square brackets account for the two different possible orientations of a feedback loop. The function $F(k, \gamma)$ is plotted in Fig. (4) as a function of $\gamma$ for different values of $k . F(k, \gamma)$ is symmetric by exchanging $\gamma$ by $1-\gamma$, corresponding to reversing the direction of the inherent directionality. Note that imposing the normalization condition $\sum_{l} A(l, k)=k !$, one can easily retrieve from Eq.(3) the probability $F(k, 1 / 2)=2^{1-k}$ in the limiting case $\gamma=1 / 2$.

The exact expression of Eq. (3) can be approximated in the asymptotic limit of large $k$ and $\gamma$ not too small (see Supplementary S6) by the expression

$$
F(k, \gamma) \approx 2 \exp \left\{\frac{k}{2} \log [\gamma(1-\gamma)]+\frac{k}{24} \log ^{2}\left(\frac{\gamma}{1-\gamma}\right)\right\} .
$$

Eq.(4) predicts that the fraction of feedback loops decays exponentially with the loop length $k$ with an amplitude factor 2 and with an exponential constant which depends on $\gamma$.

Number of ascents and cyclic Eulerian numbers. Let us consider a loop of length $k$, formed by a closed chain of $k$ nodes and $k$ edges, and let us label the nodes with numbers from 1 to $k$. We consider all the $k$ ! possible permutations of labels and aim at computing the number $A(l, k)$ of such permutations including $l$ ascents, i.e. permutations in which exactly $l$ labels in the sequence are immediately followed by a larger one. The first goal is to verify that the $A(l, k)$ 's satisfy a simple recurrence relation, similar to that obeyed by standard Eulerian numbers (see e.g. ref. 54 chapter 6 and ref. 55). To establish such a relation, let us first observe that the number of ascents does not depend on the specific ordering/permutation within a cycle. For instance the permutations 123(1), 231(2) and 312(3), which correspond to three different ways of labeling the cycle $A \rightarrow B \rightarrow C \rightarrow A$, have the same number of ascents (2, in this example). Therefore $A(l, k)=k C(l, k)$ where $C(l, k)$ corresponds to the number of ascents in the case in which the symmetry has been broken and one specific label has been chosen to be at the opening and closing extremes of the representation above. Now we look for a recurrence relation for $C(l, k)$, for which we need to express $C(l, k)$ as a function of $C(j, k-1)$, where $j=l$ or $j=l-1$. These correspond to two different cases that can occur when a new node is inserted in a loop to create a onestep larger sequence. If the node is inserted where there was an ascent, it simply replaces the previous one, so that the number of ascents remains unaltered. If it is inserted where there was a descent, a new ascent is created, so that $l$ is increased by one. These two possibilities can be summarized in the recursive equation

$$
C(l, k)=C(l, k-1) l+C(l-1, k-1)(k-1-(l-1)),
$$

where the two cases above have been weighted with the number of ascents and descents, respectively. Eq. (2) follows straightforwardly from Eq. (5) and $A(l, k)=$ $k C(l, k)$. Specific values for $k \leq 9$ obtained by iterating the recursive formula are shown in Table 2.

1. Newman, M. The structure and function of complex networks. SIAM Review 45, 167-256 (2003).

2. Albert, R. \& Barabási, A. Statistical mechanics of complex networks. Rev. Mod. Phys. 74, 47-97 (2002).

3. Barrat, A., Barthelemy, M. \& Vespignani, A. Dynamical Processes on Complex Networks (Cambridge University Press, Cambridge, 2008).

4. Arenas, A., Díaz-Guilera, A., Kurths, J., Moreno, Y. \& Zhou, C. Synchronization in complex networks. Phys. Rep. 469, 93-153 (2008).

5. Newman, M. E. J. Assortative mixing in networks. Phys. Rev. Lett. 89, 208701 (2002).

6. Alon, U. Network motifs: theory and experimental approaches. Nat. Rev. Genet. 8 , 450-461 (2007).

7. Alon, U. An Introduction to Systems Biology: Design Principles of Biological Circuits (Chapman \& Hall, London, 2006).

8. Bastolla, U. et al. The architecture of mutualistic networks minimizes competition and increases biodiversity. Nature 458, 1018-1020 (2009).

9. Johnson, S., Dominguez-garcia, V. \& Muñoz, M. Factors determining nestedness in complex networks. PloS ONE 8, e74025 (2013).

10. Girvan, M. \& Newman, M. E. J. Community structure in social and biological networks. Proc. Natl. Acad. Sci. USA 99, 7821-7826 (2002).

11. Stouffer, D. \& Bascompte, J. Compartmentalization increases food-web persistence. Proc. Natl. Acad. Sci. USA 108, 3648-3652 (2011).

12. Ravasz, E. \& Barabási, A.-L. Hierarchical organization in complex networks. Phys. Rev. E 67, 026112 (2003).

13. Corominas-Murtra, B., Goñi, J., Solé, R. V. \& Rodríguez-Caso, C. On the origins of hierarchy in complex networks. Proc. Natl. Acad. Sci. USA 103, 13316-13321 (2013).

14. Dunne, J., Williams, R. \& Martinez, N. Network structure and robustness of marine food webs. Mar. Ecol. Prog. Ser. 273, 291-302 (2004).

15. Wickens, J. \& Ulanowicz, R. On quantifying hierarchical connections in ecology. J. Social. Biol. Struct. 11, 369-378 (1988).

16. Neutel, A.-M., Heesterbeek, J. A. P. \& de Ruiter, P. C. Stability in real food webs:weak links in long loops. Science 296, 1120-1123 (2002).

17. Neutel, A.-M. et al. Reconciling complexity with stability in naturally assembling food webs. Nature 449, 599-602 (2007).

18. McCann, K. S. The diversity-stability debate. Nature 405, 228-233 (2000).

19. Allesina, S. \& Pascual, M. Network structure, predator-prey modules, and stability in large food webs. Theo. Ecol. 1, 55-64 (2008).

20. May, R. M. Food-web assembly and collapse: mathematical models and implications for conservation. Phil. Trans. R. Soc. Lond., B, Biol. Sci. 364, 1643-1646 (2009).

21. Banasek-Richter, C. et al. Complexity in quantitative food webs. Ecology $\mathbf{9 0}$, 1470-1477 (2009).

22. Sutherland, W. J. et al. Identification of 100 fundamental ecological questions. J. Ecol. 101, 58-67 (2013).

23. Mitchell, E. G. \& Neutel, A.-M. Feedback spectra of soil food webs across a complexity gradient, and the importance of three-species loops to stability. Theo. Ecol. 5, 153-159 (2012).

24. Dambacher, J. M., Luh, H. K., Li, H. W. \& Rossignol, P. A. Qualitative stability and ambiguity in model ecosystems. Am. Nat. 161, 876-888 (2003).

25. Levins, R. Discussion paper: The qualitative analysis of partially specified systems. Ann. N.Y. Acad. Sci. 231, 123-138 (1974).

26. Aigner, M. Lattice Paths and Determinants. in Computational Discrete Mathematics, vol. 2122 of Lecture Notes in Computer Science (Springer, Berlin, Heidelberg, 2001).

27. Rote, G. Path Problems in Graphs, vol. 7 of Computing Supplementum (Springer Vienna, Austria, 1990).

28. Mangan, S. \& Alon, U. Structure and function of the feed-forward loop network motif. Proc. Natl. Acad. Sci. USA 100, 11980-11985 (2003). 
29. Angeli, D., Jr., J. E. F. \& Sontag, E. D. Detection of multistability, bifurcations, and hysteresis in a large class of biological positive-feedback systems. Proc. Natl. Acad. Sci. USA 101, 1822-1827 (2004).

30. Kaufman, M., Soulé, C. \& Thomas, R. A new necessary condition on interaction graphs for multistationarity. J. Theo. Biol. 248, 675-685 (2007).

31. Plahte, E., Mestl, T. \& Omholt, S. W. Feedback loops, stability and multistationarity in dynamical systems. J. Biol. Sys. 03, 409-413 (1995).

32. Novak, B. \& Tyson, J. J. Design principles of biochemical oscillators. Nat. Rev. Mol. Cell. Biol. 9, 981-991 (2008).

33. Pigolotti, S., Krishna, S. \& Jensen, M. H. Oscillation patterns in negative feedback loops. Proc. Natl. Acad. Sci. USA 104, 6533-6537 (2007).

34. Tiana, G., Krishna, S., Pigolotti, S., Jensen, M. H. \& Sneppen, K. Oscillations and temporal signalling in cells. Phys. Biol. 4, R1 (2007).

35. Becskei, A. \& Serrano, L. Engineering stability in gene networks by autoregulation. Nature 405, 590-593 (2000).

36. Estrada, E. Characterization of 3d molecular structure. Chem. Phys. Lett. 319, 713-718 (2000).

37. Bianconi, G. \& Gulbahce, N. Algorithm for counting large directed loops. J. Phys. A: Math. Theo. 41, 224003 (2008).

38. Molloy, M. \& Reed, B. A critical point for random graphs with a given degree sequence. Random Struct. Alg. 6, 161-180 (1995).

39. G. Bianconi, N. G. \& Motter, A. E. Local structure of directed networks. Phys. Rev. Lett. 100, 118701 (2008).

40. Johnson, J. C., Borgatti, S. P., Luczkovich, J. J. \& Everett, M. G. Network role analysis in the study of food webs: An application of regular role coloration. JoSS 2, 1-15 (2001)

41. Lagomarsino, M. C., Jona, P., Bassetti, B. \& Isambert, H. Hierarchy and feedback in the evolution of the escherichia coli transcription network. Proc. Natl. Acad. Sci. USA 104, 5516-5520 (2007).

42. Yu, H. \& Gerstein, M. Genomic analysis of the hierarchical structure of regulatory networks. Proc. Natl. Acad. Sci. USA 103, 14724-14731 (2006).

43. Rodriguez-Caso, C., Corominas-Murtra, B. \& Sole, R. V. On the basic computational structure of gene regulatory networks. Mol. BioSyst. 5, 1617-1629 (2009).

44. Williams, R. J. \& Martinez, N. D. Limits to trophic levels and omnivory in complex food webs: Theory and data. Am. Nat. 163, pp. 458-468 (2004).

45. Serrano, M. A., Krioukov, D. \& ná, M. B. Self-similarity of complex networks and hidden metric spaces. Phys. Rev. Lett. 100, 078701 (2008).

46. Thieffry, D., Huerta, A. M., Pérez-Rueda, E. \& Collado-Vides, J. From specific gene regulation to genomic networks: a global analysis of transcriptional regulation in escherichia coli. Bioessays 20, 433-440 (1998)

47. Guelzim, N., Bottani, S., Bourgine, P. \& Képès, F. Topological and causal structure of the yeast transcriptional regulatory network. Nat. Genet. 31, 60-63 (2002).

48. Cohen, J. E. \& Newman, C. M. A stochastic theory of community food webs I. models and aggregated data. Proc. R. Soc. London Ser. B. 224, 421-448 (1985).
49. Williams, R. J. \& Martinez, N. D. Simple rules yield complex food webs. Nature 404, 180-183 (2000).

50. Allesina, S., Alonso, D. \& Pascual, M. A general model for food web structure. Science 320, 658-661 (2008).

51. Staniczenko, P. P. A., Smith, M. J. \& Allesina, S. Selecting food web models using normalized maximum likelihood. Meth. Ecol. Evol. 5, 551-562 (2014).

52. Ma'ayan, A. et al. Ordered cyclic motifs contribute to dynamic stability in biological and engineered networks. Proc. Natl. Acad. Sci. USA 105, 19235-19240 (2008)

53. Itzhack, R., Tsaban, L. \& Louzoun, Y. Long loops of information flow in genetic networks highlight an inherent directionality. Sys. Biom. 1, 47-54 (2013).

54. Comtet, L. Advanced Combinatorics: The Art of Finite and Infinite Expansions (Reidel Publishing Company, Dodrecht-Holland/Boston-U.S.A., 1974).

55. Graham, R., Knuth, D. \& Patashnik, O. Concrete Mathematics: A Foundation for Computer Science (Addison-Wesley, Boston, 1994).

\section{Acknowledgments}

We are grateful to $\mathrm{S}$. Johnson for a long term pleasant collaboration and to Y. Moreno for providing us with network data. We thank A. Bernacchia for suggesting the analogy with Eulerian numbers and P. Moretti and J. Hidalgo for a critical reading of the manuscript.

\section{Author contributions}

Conceived and designed the work: V.D.G., S.P. and M.A.M. Performed the experiments: V.D.G., S.P. and M.A.M. Analyzed the data: V.D.G., S.P. and M.A.M. Contributed reagents/ materials/analysis tools: V.D.G., S.P. and M.A.M. Wrote the paper: V.D.G., S.P. and M.A.M

\section{Additional information}

Supplementary information accompanies this paper at http://www.nature.com/ scientificreports

Competing financial interests: The authors declare no competing financial interests.

How to cite this article: Domínguez-García, V., Pigolotti, S. \& Muñoz, M.A. Inherent directionality explains the lack of feedback loops in empirical networks. Sci. Rep. 4, 7497; DOI:10.1038/srep07497 (2014)

This work is licensed under a Creative Commons Attribution-NonCommercialShareAlike 4.0 International License. The images or other third party material in this article are included in the article's Creative Commons license, unless indicated otherwise in the credit line; if the material is not included under the Creative Commons license, users will need to obtain permission from the license holder in order to reproduce the material. To view a copy of this license, visit http:// creativecommons.org/licenses/by-nc-sa/4.0/ 